\title{
Consideraciones sobre la génesis de una turbera meridional: la Laguna de las Madres y otras lagunas cercanas (Huelva, SW España)
}

\author{
Rocío Fernández Zamudio ${ }^{*}$, Arturo Sousa Martín ${ }^{1}$, Enrique Sánchez Gullón² \\ \& Pablo García Murillo ${ }^{1}$ \\ ${ }^{1}$ Departamento de Biología Vegetal y Ecología, Facultad de Farmacia, Universidad de Sevilla. Apdo. 874. \\ 41080 Sevilla. Spain. asousa@us.es; pgarcia@us.es; rzamudio@us.es \\ ${ }^{2}$ Paraje Natural Marismas del Odiel, Consejería de Medio Ambiente. Apdo. 720. 21080 Huelva. Spain
}

\section{RESUMEN}

En la provincia de Huelva (SO España) se encuentra una de las más importantes turberas del sur de la Península Ibérica: La Laguna de las Madres, hábitat extremadamente escaso en estas regiones meridionales. En este trabajo se muestran los resultados preliminares de un importante estudio sobre la Laguna de las Madres, actualmente en desarrollo. A partir de datos procedentes de la reconstrucción de las cuencas, del análisis de la cartografía y documentación histórica, se aportan ideas sobre la evolución y génesis de este humedal que sirve de refugio a organismos de gran singularidad con áreas de distribución reducidas o muy fragmentadas.

Palabras clave: turberas, evolución de paisajes, lagunas costeras, Huelva, SO de España.

\begin{abstract}
In the Huelva province (SW Spain) lays one of the most important peat-bogs of the southern part of the Iberian penninsula: the Laguna de las Madres, an habitat extremely rare in this meridional regions. This work shows the preliminary results of a still ongoing important study on Laguna de las Madres. rom data about the basins' reconstruction, cartography analysis and historic documents, ideas are provided about the evolution and genesis of this wetland that acts as a refuge for hughly singular organisms, with very reduced or fragmented distribution areas.
\end{abstract}

Keywords: peat bogs, landscape evolution, coastal lagoons, Huelva, SW Spain.

\section{INTRODUCCIÓN}

Las turberas son ecosistemas muy extraños en el sur de la Península Ibérica. Las turberas andaluzas se localizan en las montañas de Sierra Nevada, la depresión de Padul (Granada) y el manto eólico litoral próximo a la desembocadura del Río Tinto (Huelva): Laguna de las Madres, Rivatehilos, Peladillo (Fig. 1); también muestran carácter turboso algunos enclaves de la Sierra de Algeciras (Cádiz). Las más importantes, según los depósitos de turba que contienen, son las del Padul, con $50 \mathrm{~m}$ de potencia, y Las Madres, con cerca de 20 m (Menéndez \& Florschütz, 1964).
Tras varios años de estudio de la flora y vegetación asociada a los humedales del Manto Eólico Litoral onubense (Cirujano et al., 1998; Cobo et al., 2002; García Murillo, 2000a; 2000b, 2000c; 2001 y 2003; García Murillo \& Sousa, 1996, 1997; 1999 y 2000; García Murillo et al., 1995 y 2000; y Sousa \& García Murillo, 1998), llamó la atención la Laguna de las Madres a causa de la flora tan singular que presentaba. Más tras, al consultar material de herbario (SEV, SEVF y MA) se vio que había albergado un elevado número de elementos de carácter atlántico o eurosiberiano, muy raros en Andalucía y comunes con otras turberas del centro y norte de 


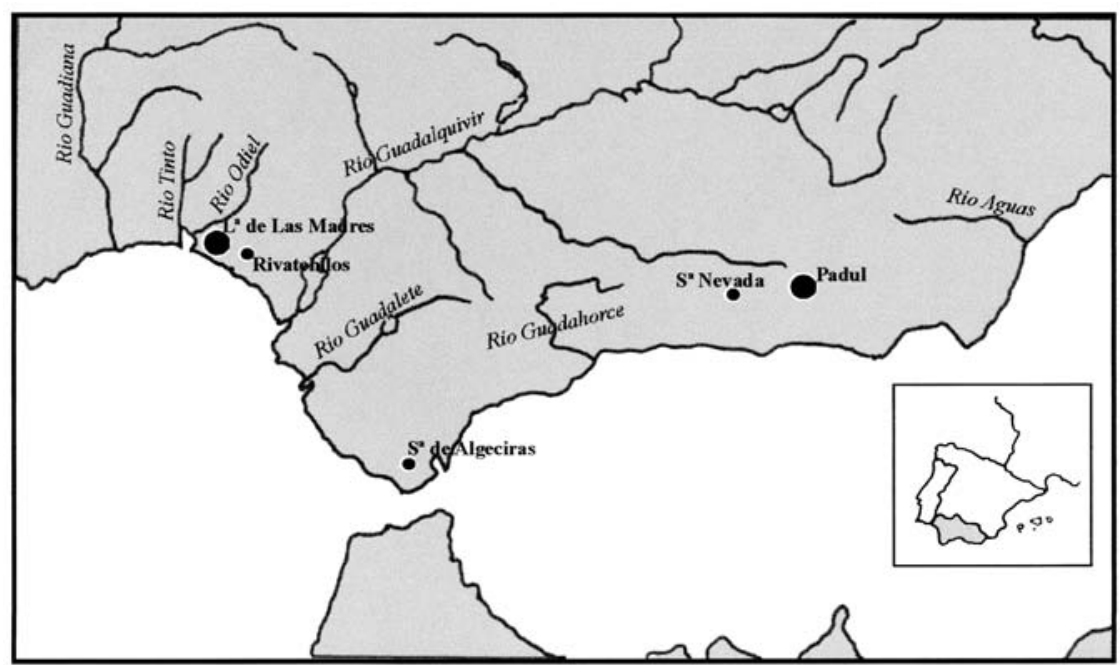

Figura 1 Importancia y distribución de los principales enclaves turbosos de Andalucía. Importance and distribution of the main peat places in Andalucia.

Europa: Carum verticillatum, Peucedanun lancifolium, Polygala vulgaris, Potamogeton polygonifolius, Sphagnum inundatum, Utricularia exoleta, etc. Asimismo se observó, con gran alarma, la gran rapidez que mostraban los procesos de deterioro de este humedal, resultado de una agresiva política agrícola desarrollada durante las dos últimas décadas, que estaba causando la extinción de la mayoría de estas especies y la substitución por otras oportunistas y banales como Phragmites australis (carrizo) o Typha dominguensis (enea) y que sin duda terminará, en pocos años, con la destrucción de la turbera.

Por todo se planteó, antes de que el deterioro fuera mayor, realizar un estudio en profundidad de la flora de este humedal y de la dinámica y cambios que habían acontecido en su vegetación y en la red de avenamiento superficial. El presente trabajo incluye los primeros resultados sobre este estudio de la Laguna de las Madres, la mayor de las turberas onubenses. En él pretendemos dar respuesta a la primera cuestión planteada: la génesis de la laguna.

Al respecto, la hipótesis vigente (Menéndez \& Florschütz, 1964) sostiene que inicialmente existirían una serie de pequeños arroyos que drenarían hacia el mar, pero que una invasión del manto arenoso fue cerrando la desembocadura de estos arroyos. Como consecuencia de ello, el dre- naje se fue dificultando hasta dejar de ser exorreico y dio lugar a la formación de un humedal (sin desagüe), como la Laguna de las Madres. Esta idea es retomada posteriormente por Zazo et al. (2000), quienes indican, además, que la Laguna de Las Madres pasó por diferentes fases, formándose a partir de una bahía marina hacia el 6500 BP. Según estos autores se convertiría en una albufera por el inicio de la barrera arenosa de Punta Arenilla, que posteriormente pasaría a formar una laguna costera como consecuencia de su aislamiento total del mar, iniciando así el proceso de acumulación de turba cerca del 4000 BP hasta cerca de 500 BP. Si esta hipótesis de Menéndez \& Florschütz (1964) es exacta, ¿cómo se explica la existencia de al menos 4 lagunas situadas en el mismo litoral, entre el Tinto y Las Madres, y que no son turbosas?, ¿por qué los arroyos próximos, pero situados más al este de la Laguna de Las Madres no han seguido este mismo proceso?

\section{ÁREA DE ESTUDIO}

El área de estudio se localiza en la provincia de Huelva, al suroeste de España, como se aprecia en la figura 1. Se trata de una zona situada en los términos de Moguer y Palos, que incluye, además de la Laguna de las Madres, un conjun- 


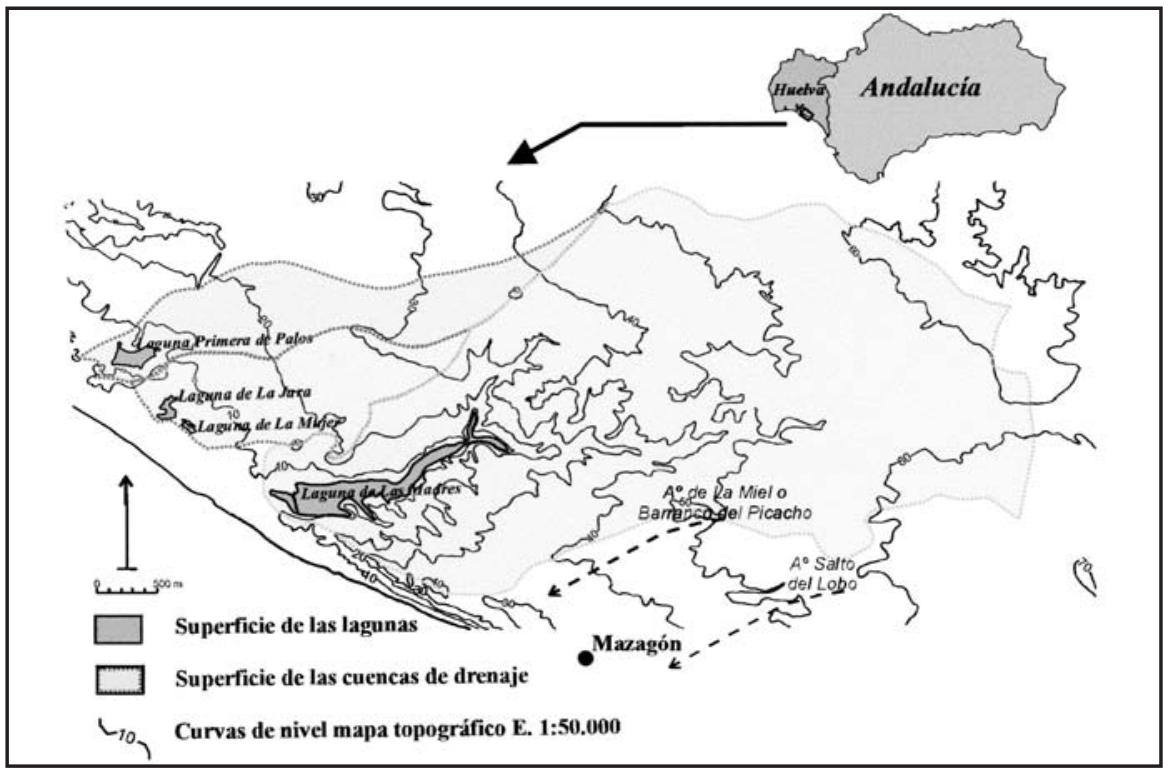

Figura 2. Área de estudio y localización de las lagunas estudiadas. Se indica también la divisoria de las cuencas de cada una de las lagunas estudiadas. Study area and localization of the studied lagoons. The basin limit of every studied lagoon is also indicated.

to de lagunas: Laguna de la Jara, Primera de Palos y de la Mujer, denominadas Complejo de lagunas de Palos. Al oeste de la Laguna de Las Madres aparecen restos de antiguos arroyos y barrancos costeros, como el Arroyo de la Miel o el Barranco del Picacho, en la actualidad casi desaparecidos por el crecimiento urbanístico de las proximidades de Mazagón, y la colmatación de sus cauces por las arenas (Fig. 2).

El complejo lagunar de Palos y Las Madres se desarrolla sobre el manto eólico litoral onubense (Pastor \& Leyva, 1976), y en la actualidad aparece rodeado de una gran cantidad de cultivos forzados bajo plástico. Respecto a las carac- terísticas climáticas, la zona presenta un clima mediterráneo con influencia oceánica. La precipitación media anual para la estación meteorológica de Moguer es de 534 mm/año (para el período 1970-2000, de acuerdo a los datos del Instituto Nacional de Meteorología).

Todas estas lagunas aparecen conformando el Paraje Natural de las Lagunas de Palos y las Madres, con una superficie protegida de 693 ha (Consejería de Medio Ambiente, 2001), contempladas por la Ley 2/1989 que protege los espacios naturales de Andalucía, y propuestas como Lugar de Interés Comunitario (LIC), de acuerdo a la Directiva Hábitats.

Tabla 1. Fuentes de datos para la reconstrucción de los sistemas palustres de la zona de estudio. Data sources for the reconstruction of the marshy systems in the study area.

\begin{tabular}{lccc}
\hline & & \multicolumn{2}{c}{ Fuente de datos } \\
\cline { 2 - 4 } Período temporal & Fotografía aérea & Archivos y cartografía histórica & Interpolación del microrelieve \\
\hline Estado original $(\cong$ S. XVII) & No & Sí & Sí \\
Finales del S. XIX & No & Sí & Sí \\
$\mathbf{1 9 5 6}$ & Vuelo B/N E. 1:33.000 & No & No \\
$\mathbf{1 9 8 7}$ & Vuelo B/N E. 1:20.000 & No & No \\
$\mathbf{2 0 0 0}$ & Vuelo B/N E. 1:30.000 & No & No \\
\hline
\end{tabular}

* Ver las fuentes cartográficas utilizadas en el Anexo Cartografía Histórica Consultada 


\section{METODOLOGÍA}

Para responder a las cuestiones apuntadas en la Introducción se han utilizado tres métodos además de prospecciones "in situ" para reconocer la flora y vegetación del lugar.

1. Se ha elaborado un mapa hipsométrico de la zona de estudio con curvas de nivel cada $2 \mathrm{~m}$ mediante la interpolación de curvas de nivel del Mapa Topográfico de Andalucía, E. 1:10.000 (1987), a partir de más de 500 cotas topográficas, siguiendo el método descrito por Sousa \& García Murillo (2002). El mismo método se empleó para la reconstrucción del cauce de los arroyos desde el S. XVII al XX, utilizando el mismo mapa, así como el material indicado en la Tabla 1. A partir de estos mapas hipsométricos detallados, también se delinearon las divisorias de aguas que separan las diferentes cuencas drenaje de cada una de las lagunas estudiadas.

2. Se tomaron medidas sobre la superficie y longitud de los arroyos y de las lagunas, todas ellas con un planímetro PLANIX 5000, sobre los mapas topográficos a E. 1:10.000 del Instituto Andaluz de Cartografía, para establecer la morfometría de estas zonas húmedas y de sus cuencas.

3. Finalmente se hizo un análisis de las descripciones antiguas de la zona contenidas en distintos documentos históricos como el Catastro de La Ensenada, S. XVII (Gómez Cruz, 1991), las Relaciones de Tomás López, S. XVIII (Ruiz González, 1999), el
Diccionario Geográfico-Estadístico de Miñano (1826), el Diccionario Geográficoestadístico-histórico de España y sus posesiones de Ultramar de Madoz (1848), y varios documentos del archivo histórico municipal de Moguer, así como de la cartografía histórica referida en el Anexo. El período histórico abarcado en esta revisión corresponde a los S. XVII al XX.

Igualmente, para establecer si existía alguna relación entre la superficie que ocupan las cuencas de cada uno de los arroyos y la longitud original de éstos, se calculó el Coeficiente de Correlación de Pearson. Para aumentar el tamaño de muestra, este cálculo se realizó incluyendo todos los arroyos existentes entre la Torre del Loro hasta la Laguna de las Madres (11 en total). Dado que el tamaño de muestra sigue siendo bajo se calculó también el coeficiente de correlación para un test no paramétrico (Coeficiente de Spearman).

\section{RESULTADOS}

Las observaciones sobre la flora y vegetación de las lagunas de las Madres y las del Complejo de Palos muestran marcadas diferencias.

En la primera, en algunas zonas, aparece bien desarrollada una orla leñosa caracterizada por la presencia de Erica ciliaris, que es acompañada por Ulex minor, Cistus psilosepalus, Molinia caerulea y Genista ancystrocarpa, comunidad típica de suelos turbosos hidromorfos. Asimismo

Tabla 2. Características morfométricas de las lagunas de Palos y Las Madres. Morphometric characteristics of Palos and Las Madres lagoons.

\begin{tabular}{|c|c|c|c|c|c|}
\hline & $\begin{array}{l}\text { Superficie } \\
\text { cuenca (ha) }\end{array}$ & $\begin{array}{c}\text { Superficie } \\
\text { laguna }(\mathbf{k m})\end{array}$ & $\begin{array}{l}\text { Anchura mínima } \\
\text { laguna }(\mathbf{k m})\end{array}$ & $\begin{array}{l}\text { Anchura máxima } \\
\text { laguna }(\mathbf{k m})\end{array}$ & $\begin{array}{c}\text { Longitud máxima } \\
\text { laguna línea recta }(\mathrm{km})\end{array}$ \\
\hline$L^{a}$ Primera de Palos & 499.3 & 10.2 & 0.17 & 0.30 & 0.57 \\
\hline$L^{a}$ de la Jara & - & 3.7 & 0.05 & 0.27 & 0.29 \\
\hline$L^{a}$ de la Mujer & - & 1.2 & 0.03 & 0.11 & 0.21 \\
\hline$L^{a}$ de la Jara y $L^{a}$ de la Mujer & $448.9 *$ & 4.9 & - & - & - \\
\hline$L^{a}$ de las Madres & 3337.4 & 60.1 & 0.10 & 0.43 & 2.66 \\
\hline
\end{tabular}

* Las lagunas de la Jara y de La Mujer tienen una cuenca hidrográfica común, por lo que aparece así reflejada la medida. 
Retroceso de los arroyos costeros

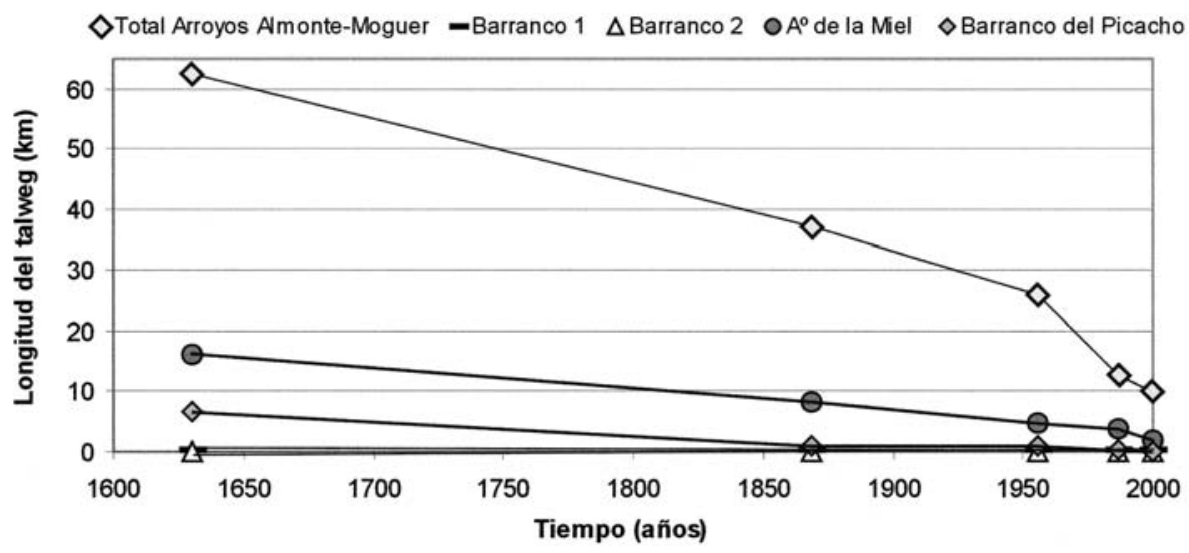

Figura 3. Evolución de la longitud de los arroyos y barrancos costeros desde el S. XVII al XX. Evolution of the length of streams and coastal gullies length since $17^{\text {th }}$ to $20^{\text {th }}$ century.

la vegetación helofítica está, en general, bien desarrollada; con notables manchas de Cladium mariscus (especie muy poco común en Andalucía) y, en las zonas más alteradas, Phragmites australis; además de dos especies de Typha: T. latifolia y $T$. dominguensis. La vegetación sumergida es muy escasa apareciendo puntualmente poblaciones de Potamogeton polygonifolius, Nymphaea alba y Polygonum amphybium.

En cambio las lagunas del complejo de Palos muestran una escasísima orla de vegetación, en ella no se encuentran elementos leñosos y las únicas especies que se observan son los helófitos: Phragmites australis y Typha dominguensis. Los macrófitos sumergidos, a excepción de la Laguna Primera de Palos, cuya cubeta está toda ella cubierta de Ceratophyllum submersum, están escasamente representados, habiéndose observado las especies: Zannichellia obtusifolia, Potamogeton pectinatus y Chara connivens.

Los datos morfométricos de las lagunas medidos en la cartografía actual (Mapa Topográfico de Andalucía, E. 1:10.000, 1987) y en la histórica, se recogen en la Tabla 2, donde destaca que la superficie ocupada por la cuenca de drenaje de Las Madres es muy superior a la ocupada por el Complejo de las lagunas de Palos.

El estudio diacrónico de la longitud de los cauces de los arroyos costeros apunta que estos han sufrido una importante regresión, que ha supuesto hasta un $84 \%$ de la longitud. La figura 3 representa estas observaciones. Asimismo, se ha relacionado la longitud original de los arroyos y barrancos costeros con la superficie que ocupa cada una de las cuencas de drenaje correspondientes. El resultado aparece en la figura 4 , a la que se ha agregado la relación lineal representando la línea de tendencia:

Aunque el tamaño de muestra no es óptimo (dado que sólo existen 11 arroyos y barrancos en la zona), el análisis de correlación muestra una tendencia clara como refrenda el hecho de que la R-SQ sea muy alto (casi 0.9). Como test

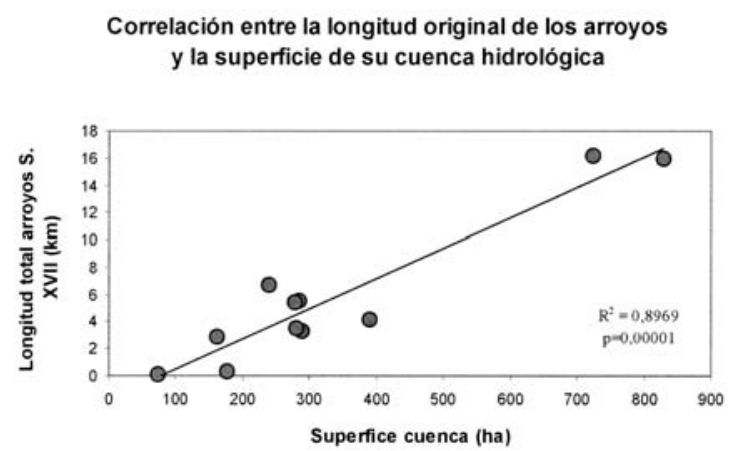

Figura 4. Relación entre la longitud de los arroyos del litoral de Almonte y Moguer y la superficie ocupada por su cuenca de drenaje. Relationship between the coastal streams length and their watershed surface. 
no paramétrico se ha calculado también el Coeficiente de Correlación de Spearman, que también muestra una fuerte relación entre las variables $(r=0.7272, p=0.0112)$.

En el análisis de las descripciones y cartografía histórica de la zona de estudio destaca el hecho de que en determinados mapas de la época aparezcan con frecuencia conectadas en una vaguada común las lagunas de Palos con la zona más meridional de Las Madres, conformando una vaguada o zona húmeda común. Este es el caso de la cartografía de Coello (1869), Carrasco Padilla (1892), y de manera más difusa aparece también en Fernández y Coria (1862), Montojo y Salcedo (1875), Gonzalo y Tarín (1887) y San Miguel (1913). Detalles de ello se pueden apreciar en las figuras 5 y 6.

Para profundizar en este aspecto se realizó la interpolación del relieve en base a las cotas topo- gráficas existentes en los mapas E. 1:10.000 (Mapa Topográfico de Andalucía, E. 1:10.000, 1987), los resultados mostraron:

- Las lagunas de la Jara y de la Mujer -que como se explicó previamente comparten una misma cuenca hidrográfica- presentan una cubeta común, que incluye a las lagunas actuales, en una cota aproximada de $6 \mathrm{~m}$.

- Aunque las cubetas aparecen separadas entre si, todas se incluyen en una vaguada común (Primera de Palos Jara, Mujer y Las Madres), aproximadamente sobre la cota $10 \mathrm{~m}$.

También, se observó en diversa cartografía histórica la presencia de un pequeño canal (de poco más de $200 \mathrm{~m}$ ) que conectaba la Laguna de Las Madres y el océano. Este canal se localizaría en el borde más suroccidental de Las

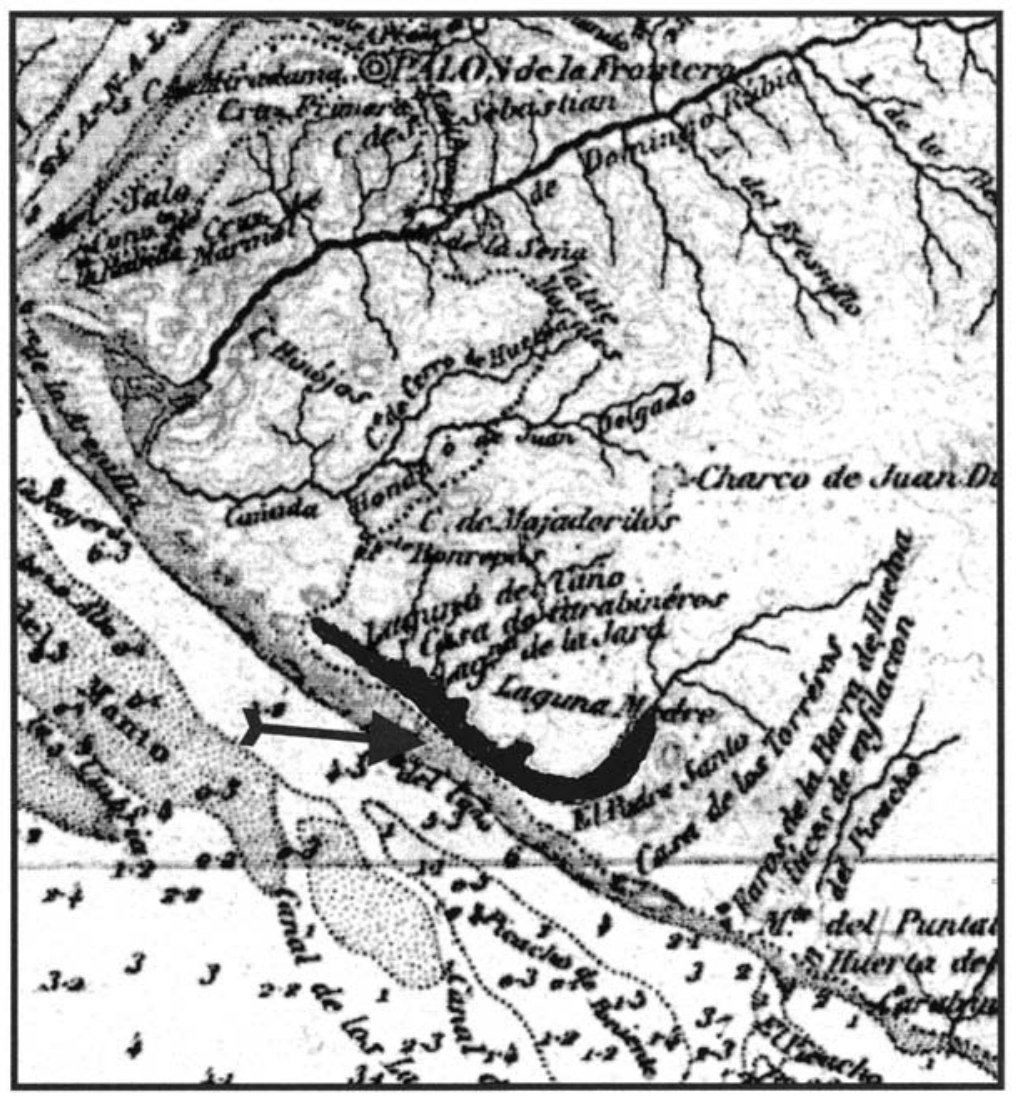

Figura 5. La cartografía de Coello (1869) muestra la conexión entre las lagunas de Palos y la Las Madres, destacadas con una flecha negra. Coello's cartography (1869), shows the connexion between Palos and Las Madres lagoons marked with a black arrow. 
Madres y aparece ya a finales del S. XIX recogido en diversa cartografía Anónimo (S. XIX), Carrasco Padilla (1892), Instituto Geográfico y Estadístico (1987-1900), Noriega y Cobo del Guzmán (1897-1900). En la figura 7 se puede ver un detalle de ello.

Finalmente, resulta también muy llamativa la descripción que hace Gonzalo y Tarín (1886) sobre la zona de Las Madres conocida con el sugerente topónimo de Las Tembladeras. La voz Tembladeras es equivalente a la de Tremedal o a Tembledal, que según González Bernáldez (1992) hace referencia a la sensación de "temblar" que se produce al caminar sobre la turba:

"Algunos Km. al norte de la laguna se encuentran los manantiales denominados Las Madres de Moguer, á donde fluye el agua depo- sitada en el extenso manto arenoso de que hablamos antes. Desde aquel punto siguen el curso que les marca una cañada con fondo de arena en que paulatinamente se va perdiendo por filtración, hasta que desaparecen por completo, siguiendo, sin embargo, en corriente subterránea, hasta el sitio conocido por Las Tembladeras, en donde surge otra vez á la superficie para precipitarse luego, á los pocos metros de distancia, (unos 4), con bastante estrépito, por otro agujero, siguiendo después subterráneamente hasta la referida laguna" (sic).

Esta situación en el drenaje superficial y subterráneo de la Laguna a finales del S. XIX, contrasta con la situación actual y describe un funcionamiento en un período donde la disponibilidad de agua era mayor y la humedad edáfica mucho más alta.

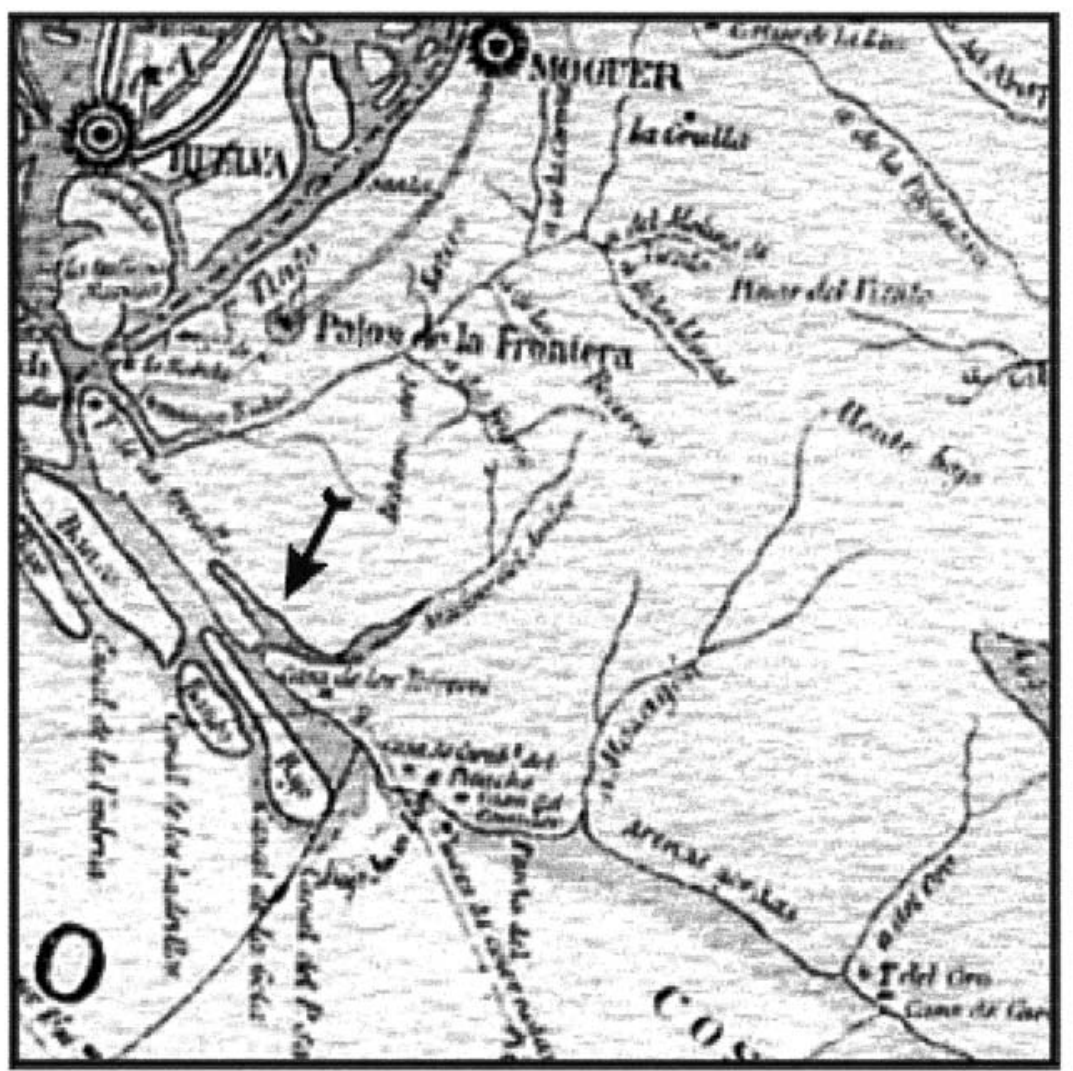

Figura 6. Detalle de la cartografía de Carrasco y Padilla (1892), donde se aprecia también la conexión de las lagunas de Palos entre sí y con la L de Las Madres, y además se marca con una flecha negra el canal que conectaba esta con el Océano Atlántico. Detail from the cartography of Carrasco and Padilla (1892), the connexion between Palos and Las Madres lagoons can be noticed. In addition, the union between this lagoon and the ocean is marked with a black arrow in the map. 


\section{DISCUSIÓN}

Los datos morfométricos obtenidos, procedentes de la interpolación del relieve y del análisis de la cartografía histórica, ponen en evidencia la existencia de un antiguo complejo de lagunas cercano a la Laguna de las Madres, muy relacionado con ésta y con una vaguada común. Dicho complejo, además de la laguna de las Madres, incluía: la Laguna Primera de Palos, la Laguna de la Jara, la Laguna de la Mujer y la Laguna de los Barreros, con un aspecto y unas características muy distintas a la que presenta la Laguna de las Madres. Algunas de estas, como la Laguna de los Barreros, en la actualidad, han desaparecido tras las obras de construcción del polo químico de Huelva.

Si se toma como idea de partida que la acumulación de turba en la Laguna de las Madres se produjo como consecuencia de la obturación de su drenaje al Océano Atlántico (Menéndez \& Florschütz, 1964); tanto los restos del Arroyo que aparecen en la cartografía reciente (Mapa de Andalucía; 1987) próximos al complejo de lagunas de Palos (que ocupan longitudes entre 1 y $4.6 \mathrm{Km}$, según la laguna de que se trate), como algunas representaciones observadas en la cartografía histórica consultada [donde aparecen más desarrollados estos afluentes que drenaban hacia las lagunas (Coello, 1869)], permiten pensar que el origen para estas lagunas es el mismo que el indicado por Menéndez \& Florschütz (1964) para la Laguna de las Madres.

Por lo tanto, cabe la posibilidad de que las diferencias entre la Laguna de las Madres y las del Complejo de Palos, relativas a su aspecto, flora y vegetación puedan deberse a

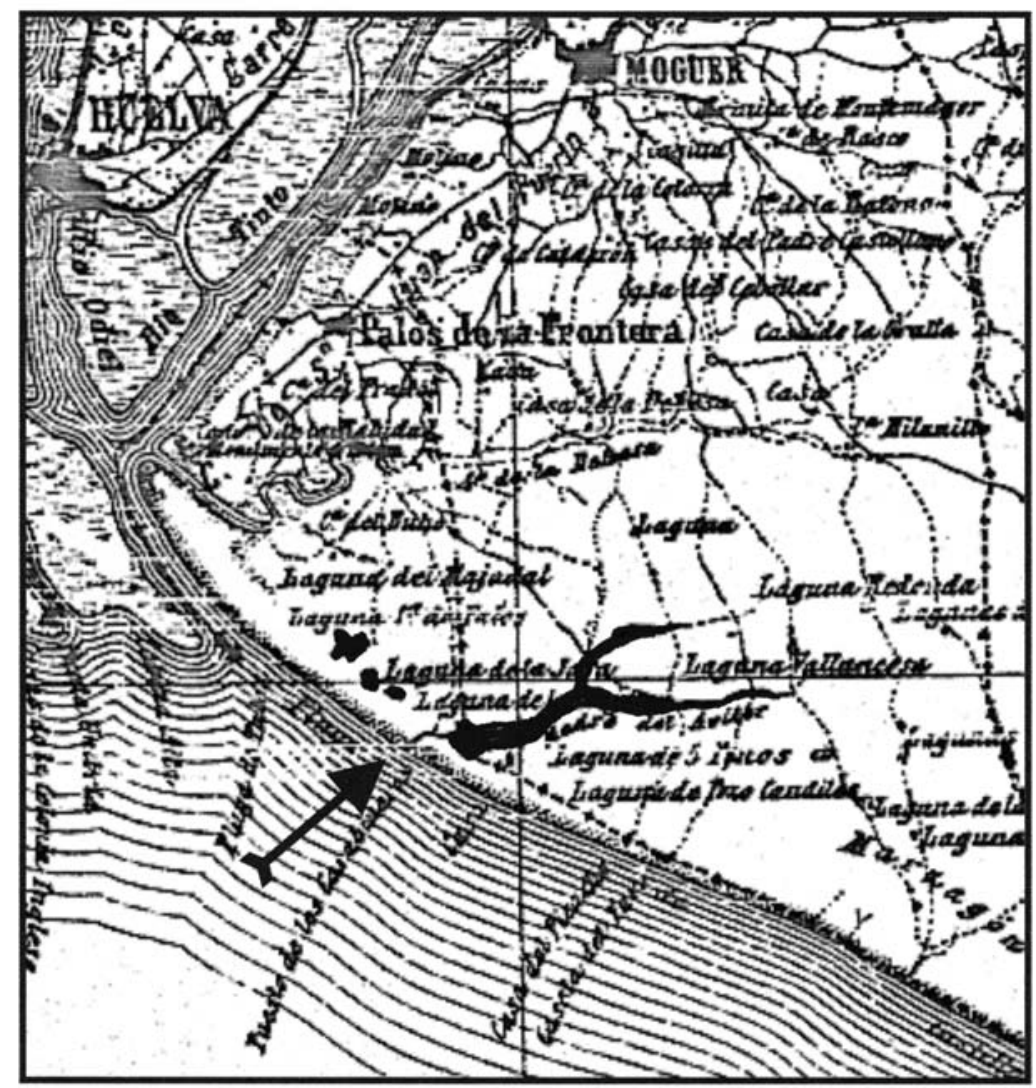

Figura 7. Detalle de la cartografía de Noriega y Cobo del Guzmán (1897-1900), donde se aprecia la conexión entre el océano y la laguna turbosa de Las Madres. Detail from the cartography of Noriega and Cobo de Guzman (1897-1900), where the connexion between the ocean and Las Madres peat-bog can be seen . 
las características de las cuencas y/o a sus diferentes estadíos evolutivos.

La cuenca del conjunto de las lagunas del Complejo de Palos es mucho menor que la de las Madres (ver tab. 2), como consecuencia, los procesos de colmatación del talweg se sucederían con mayor rapidez.

Asimismo, al tratarse de un episodio de colmatación mucho más rápido, cabe pensar que las lagunas del Complejo de Palos se encontrarían en un estadío evolutivo mucho más avanzado y de mayor senectud que el de la Laguna de las Madres. En este sentido, es posible que la obturación de los cauces de los arroyos originales, se diera de manera simultánea, si bien, con un ligero desfase que consistiría en un retraso en el proceso en los arroyos situados más al este. De forma que la obturación se haría por este orden: Laguna de Barreros, después en Primera de Palos, después en La Jara, luego en La Mujer y finalmente en la Laguna de las Madres.

De tal manera que, teniendo en cuenta estos factores, dispondríamos de una cuenca 6 veces mayor en la Laguna de las Madres que en el Complejo de Palos. Esta mayor cuenca aportaría una mayor alimentación hídrica y una mayor cantidad de materia orgánica para la formación de horizontes turbosos.

También hay que señalar la existencia de una serie de barrancos litorales, situados al este de la Laguna de las Madres que corresponden a antiguos arroyos, y que muestran también una apariencia distinta de la de los arroyos hasta ahora señalados. ¿Por qué no han seguido el mismo proceso evolutivo que los otros arroyos dando lugar a la formación de lagunas litorales como las del Complejo de Palos o Las Madres?

Los datos que hemos obtenido de la evolución de los dos barrancos más próximos al este de la Laguna de las Madres: Arroyo de la Miel y Barranco del Picacho, muestran características similares a las ya observadas en los arroyos del Parque Natural de Doñana, muy próximos y estudiados por Sousa \& García Murillo (2002 y 2003). En nuestra opinión, la evolución diferenciada de estos arroyos procede de estar sometidos a una diferente dinámica litoral. De acuerdo con Ojeda Zujar (1988), la influencia de la desembocadura del Río Guadiana se hace sentir hasta un poco más allá de la Desembocadura del Río Tinto (Laguna de las Madres), en cambio desde Mazagón hasta la desembocadura del Guadalquivir (donde se sitúan los arroyos de la Miel y Barranco del Picacho) hay un ángulo de mayor eficacia en de la deriva litoral de la costa, que acentua los procesos erosivos y que ha quedado reflejado en el retroceso del Acantilado costero del Asperillo, próximo a Mazagón, y de otros médanos adyacentes (San Miguel, 1913; Vanney \& Menateau, 1979). Por tanto, puesto que es una zona de erosión, no se produce la acumulación o movilización de un manto arenoso que obture la desembocadura.

Este diferente comportamiento explicaría la razón por que estos arroyos no habrían evolucionado hacia formaciones lagunares litorales como es el caso de las Lagunas del Complejo de Palos y Las Madres. Y es que, como señalan Fernández Palacios et al. (1990), la convergencia de la capacidad de arrastre de la derivas litoral oeste-este, junto con la acumulación de arrastres fluviales en las mismas desembocaduras, genera barras o flechas arenosas que tienden a cerrar los estuarios de forma total, generando lagunas prelitorales como Las Madres.

También, apuntar que la hipótesis sostenida por Sousa \& García Murillo (2002 y 2003) de que la Pequeña Edad del Hielo, que Pita (1997) data desde 1430 a 1850 en el litoral onubense, fue un período con una mayor atlanticidad en el clima, encaja con los datos obtenidos de microtopografía, cartografía histórica y antiguas descripciones; como las referidas por Gonzalo y Tarín (1887), relativas al complejo Laguna de las Madres -Lagunas de Palos. Es evidente que durante este período, en las épocas de mayor pluviosidad, todas las lagunas del Complejo estuvieran conectadas, aunque fuera de manera temporal, dando origen así a la vaguada común referida anteriormente.

En definitiva, los datos derivados de la cartografía y las fuentes documentales históricas reflejan unas condiciones, situadas varios siglos atrás, de mayor encharcamiento ( $\mathrm{y}$ a veces de mayor 
interconexión) entre las lagunas del Complejo de Palos y Las Madres, los cuales coinciden con los obtenidos mediante un análisis detallado del relieve las cuencas de las mencionadas lagunas. Todo ello apunta a unas circunstancias climáticas pretéritas más benignas, que permitieron el desarrollo de una vegetación de marcado carácter atlántico y de la cual quedan algunos enclaves y elementos de naturaleza relicta y singular.

De igual forma, la evolución diferencial de los arroyos que dieron lugar a estas lagunas litorales del Complejo de Palos-Las Madres y los que se encuentran al este de estas, parece deberse a una dinámica litoral diferenciada entre ambos sectores costeros.

Finalmente, resaltar la importancia de la conservación de este complejo lagunar, que además de constituir un humedal con una flora excepcional, sirve para ilustrar los procesos de cambios ambientales que recientemente ha sufrido el litoral onubense y los factores, que a nivel global, han intervenido en él.

\section{AGRADECIMIENTOS}

Los autores desean agradecer al Director del Paraje Natural, Dr. D. Juan Carlos Rubio, las facilidades dadas par la realización de este trabajo.

\section{BIBLIOGRAFÍA}

COBO, M. D., E. SÁNCHEZ-GULÓN \& P. GARCÍA MURILLO. 2002. Flora y Vegetación. En: Canseco et al. (eds.) Parque Nacional de Doñana. Canseco Eds. SL: Talavera de la Reina. pp. 109-174.

CONSEJERÍA DE MEDIO AMBIENTE. 2001. Medio Ambiente en Andalucia. Informe 2000. Consejería de Medio Ambiente, Junta de Andalucía. Sevilla, 506 pp.

CIRUJANO, S., L. MEDINA, P. GARCÍA MURILLO \& J. L. ESPINAR. 1998. Ricciocarpus natans (L.) Corda (Ricciaceae) en la Península Ibérica. Anales J. Bot., 56: 366-368.

FERNÁNDEZ-PALACIOS, J. M., M. J. MARTOS \& J. C. RUBIO. 1990. Las marismas atlánticas andaluzas. Quercus, 54: 15-22.
GARCIA MURILLO, P. 2000a. Hydrocharis morsus-ranae. En: Libro Rojo de la flora silvestre amenazada de Andalucía. Vol. II. B. Valdés et al. (eds.): 173-177. Consejería de Medio Ambiente. Junta de Andalucía. Sevilla.

GARCÍA MURILLO, P. 2000b. Utricularia exoleta. En: Libro Rojo de la flora silvestre amenazada de Andalucía. Vol. II. Valdés et al. (eds.): 361-363. Consejería de Medio Ambiente. Junta de Andalucía. Sevilla.

GARCÍA MURILLO, P. 2000c. Wolffia arhiza. En: Libro Rojo de la flora silvestre amenazada de Andalucía. Vol. II. Valdés et al. (eds.): 373-375. Consejería de Medio Ambiente. Junta de Andalucía. Sevilla.

GARCÍA MURILLO, P. 2001. Restauración del complejo Palustre del Abalario: la reconstrucción del Paisaje. En: $1^{a}$ Reunión Internacional de expertos sobre la regeneración hídrica de Doñana. Ponencias y Conclusiones. Ministerio de Medio Ambiente (ed.) Ministerio de Medio Ambiente. Madrid. pp. 117-130.

GARCÍA MURILLO, P. 2003. Plantas acuáticas y carnívoras en Doñana. Doñana 2005, 4: 8-11.

GARCÍA MURILLO, P. \& A. SOUSA. 1996. Vulpia fontquerana Melderis \& Stace (Gramineae) ¿Endemismo Gaditano-Onubense? Anales J. Bot., 55: 174-176.

GARCÍA MURILlO, P. \& A. SOUSA. 1997. Vegetation changes in: Abalario (Natural Park of Entorno de Doñana, SW Spain). Lagascalia, 19: 737-744.

GARCÍA MURILLO, P. \& A. SOUSA. 1999. El paisaje vegetal de la zona oeste del Parque Natural de Doñana (Huelva). Lagascalia, 21: 111-132.

GARCÍA MURILLO, P. \& A. SOUSA. 2000. Vulpia fontquerana. En: Libro Rojo de la flora silvestre amenazada de Andalucía. Vol. I. Valdés et al. (eds.): 298-309. Consejería de Medio Ambiente. Junta de Andalucía. Sevilla.

GARCÍA MURILLO, P., A. SOUSA \& E. FUERTES. 1995. Sphagnum inundatum, nuevo para Andalucía. Anales J. Bot., 53:245.

GARCÍA MURILLO, P., S. CIRUJANO, L. MEDINA \& A. SOUSA. 2000. ¿Se extinguirá Hydrocharis morsas-ranae L. de la Península Ibérica. Portugaliae Acta Biologica, 19: 149-158.

GÓMEZ CRUZ, M. 1991. Atlas histórico forestal de Andalucía del S. XVIII. Servicio de $\mathrm{Pu}-$ blicaciones de la Universidad de Granada. Granada. $71 \mathrm{pp}$. 
GONZALEZ BERNALDEZ, F. 1992. Los Paisajes del Agua. Terminología popular de los Humedales. Reyero. Madrid. 257 pp.

GONZALO Y TARÍN, J. 1887. Mapa geológico y topográfico de la provincia de Huelva. Escala 1: 400.000 y memoria. Inst. Geológico y Minero de España. Madrid.

MAPA TOPOGRÁFICO DE ANDALUCÍA. 1987. Provincia de Huelva. 1:10000. Mosaíco Raster. Instituto de Cartografía de Andalucía. Consejería de Obras Públicas y Transportes. Junta de Andalucía. Soporte CD ROM.

MADOZ, P. 1848. Diccionario Geográfico-estadistico-histórico de España y sus posesiones de Ultramar. Edición facsímil de las voces de la provincia de Huelva. Sánchez Zorro, D. (ed) . Ámbito Ediciones (reimpresión 1988). Salamanca. 132 pp.

MENÉNDEZ, J. \& F. FLORSCHÜTZ. 1964. Resultados del análisis paleobotánico de una capa de turba en las cercanía de Huelva (Andalucía). Estudios Geológicos, XX: 183-186.

MIÑANO, S. 1826. Diccionario Geográfico Estadistico de España y Portugal. Imprenta de PierartPeralta. Madrid.

OJEDA ZÚJAR, J. 1988. Peculiaridades morfodinámicas de la fachada ibérica del Golfo de Cádiz: geomorfología litoral. Revista de Estudios Andaluces, 10: 53-68.

PASTOR, F. \& F. LEYVA. 1976. Mapa Geológico Nacional. Hoja 1000 “Moguer". Memoria y mapa 1:50.000. Inst. Geológico y Minero de España. Madrid.

PITA, M. F. 1997. Los cambios climáticos. En: Climatología. Cuadrat, J. Mª \& Pita, M. F. (eds.): 387-481. Cátedra. Madrid.
RUIZ GONZÁLEZ, J. E. 1999. Huelva, según las relaciones enviadas por los párrocos al geógrafo real Tomás López en el siglo XVIII. Edición y recopilación original del S. XVIII. Diputación Provincial de Huelva. Huelva. 427 pp.

SAN MIGUEL, M. 1913. Las costas de la provincia de Huelva y sus variaciones en el periodo histórico. Bol. R. Soc. Esp. Hist. Nat., XIII: 434468.

SOUSA, A. \& P. GARCÍA MURILLO. 1998. Cambios históricos en el avenamiento superficial y la vegetación del Parque Natural del Entorno de Doñana (Sector Abalario, Huelva). Ería, 46: 165-182.

SOUSA, A. \& P. GARCÍA MURILLO. 2002. Méthodologie pour l'étude des effets du Petit Age Glaciaire dans le Parc Naturel de Doñana (Huelva, Espagne). Essai de reconstitution des formations palustres et du drainage superficiel. Publication de l'Association Internationale de Climatologie, 14: 359-367

SOUSA, A. \& P. GARCÍA-MURILLO. 2003. Changes in the wetlands of Andalusia (Doñana National Park, SW Spain) at the end of the Little Ice Age. Clim. Change, 58: 193-213

VANNEY, J. R. \& L. MENANTEAU. 1979. Types de reliefs littoraux et dunaires en basse Andalusie (de la ría de Huelva a l'embouchure du Guadalquivir). Melanges de la Casa Velásquez, 15: 1-52.

ZAZO, C., F. BORJA, F. DÍAZ DEL OLMO, C. J. DABRIO, J. L. GOY \& A. C. STEVENSON. 2000. Laguna de las Madres. En: Envionmental Changes during the Holocene. Fieldtrip Guide: Litoral de Huelva. C. Zazo, F. Borja, F. Díaz del Olmo, C. J. Dabrio, J. L. Goy, A. C. Stevenson \& C. Gómez (eds): 42-53. INQUA Comisión on the Holoceno. Sevilla. 


\section{ANEXO I}

\section{CARTOGRAFÍA HISTÓRICA CONSULTADA}

1606. “Andalucía Nova”. Autor: Jodocus Hondius. Cartoteca Instituto Geográfico Nacional.

1640-1650. “Andaluzia continens Sevillam et Cordobam”. Autor: Willem Blaeu. Escala: 1.333.333. Theatrum du Monde au Nouvel Atlas. Cartoteca Instituto Geográfico Nacional.

1696. “Li Regni de Granata E D’Andalucia”". Autor: Domenico de Rossi (Domenicus Rubeis). Cartoteca Instituto Geográfico Nacional.

S. XVII. “Mapa de España de Carlos III”. Autor: J. Danckaert. Theatre de la Guerre de Espagne et en Portugal, Preferente a Charles III. Roy d'Espagne et des Indes, etc: etc: a Amsterdam. C. Danckerts avec Privilege (sic). Accuratis sima, totius Regni, Hispania, Tabula Per Instinum Danckaerts (sic). Cartoteca Instituto Geográfico Nacional.

1781. “Carte de Seville”. Autor: F. L. Gussefeld. Imp. Herederos de Homann. Escala. 1:623.000. Escala gráfica en leguas de Alemania (7.409 m.), castellanas legales $(5.560 \mathrm{~m}$.) y de una hora de camino $(6.620 \mathrm{~m}$.), de 20 al grado y toscas (1,9 m.). Cartoteca Instituto Geográfico Nacional.

1810. "Plano geográfico de Moguer, con los movimientos del General Lacy”, por D. Angel Saavedra, Ayudante Primero de E. M. Plano Geográfico de Moguer y sus inmediaciones, referentes a los movimientos de la División General Laci en Agosto de 1810 quando batió a las tropas del general ArambergoAtula de León y Septiembre primero de 1810= Mampoey=(sic). Escala aprox. 1:150000 (estimada). Escala gráfica en leguas. Sin proyección alguna. Perfiles abatidos. Cartoteca Histórica del Servicio Geográfico del Ejército.

1862. "Plano de los ríos Tinto y Odiel”. Autor: Manuel Fernández y Coria. Dirección de hidrografía, Madrid. Escala 1:25000. En: "La Representación del territorio, Huelva”. Cartoteca del Instituto Geográfico Nacional.

1869. "Huelva”, por D. Francisco Coello, Coronel de Ingenieros. Atlas de España y sus posesiones de ultramar. En: Diccionario Geográfico-Estadístico-Histórico. Notas estadísticas e históricas escritas por Pascual Madoz. 1869, Madrid. Escalas gráficas: Leguas legales, Millas Marítimas, etc. Escala aprox. 1:200.000. Meridiano de origen de Madrid. Cartoteca Histórica del Servicio Geográfico del Ejército.

1875. “Costa Sudoeste de España”.Hoja II (desde Huelva hasta la Torre de la Higuera). Según trabajos realizados desde 1865-1870 por la comisión Hidrográfica a cargo de D. José Montojo y Salcedo. Dirección de Hidrografía, Madrid, 1875. Carta náutica. Escala aprox. 1:50.000. Meridiano en el origen de San Fernando. Sin escala gráfica. Cartoteca Histórica del Servicio Geográfico del Ejército.

1880. Provincia de Huelva”, por D. Emilio Valverde, Comandante de Infantería. Escala: 1:750.000. Atlas Geográfico descriptivo de la Península Ibérica, Islas Baleares, Canarias y Posesiones Españolas en Ultramar por el Comandante graduado Capitán de Infantería D. Emilio Valverde y Alvarez, auxiliar que ha sido del depósito de los Guerra, Provincia de Huelva (sic). Imprenta y fotografía de la Biblioteca Nacional. Cartoteca Histórica del Servicio Geográfico del Ejército. Sin escala gráfica ni proyección.

1887. "Mapa geológico y topográfico de la provincia de Huelva”, por D. Joaquín Gonzalo y Tarín. Escala 1:400.000. Biblioteca Instituto Geológico y Minero.

1892. “Nuevo mapa geográfico estadístico de la provincia de Huelva”. Autor: José Carrasco Padilla (Ecxma. Diputación Provincial de Huelva). Escala: 1: 300.000. Meridiano de origen de Madrid. En "La representación del territorio, Huelva". Cartoteca del Instituto Geográfico Nacional.

1897-1900. "Provincia de Huelva, nivelación”, por el Instituto Geográfico y Estadístico. Dibujado por F. Noriega y J. Cobo de Guzmán. Trabajos topográficos desde 1897 á 1900. Proyección Tissot. Altimetría deducida del mapa 1:100.000. Sin escala gráfica, meridiano de origen de Madrid. Escala estimada 1:500.000. Cartoteca Histórica del Servicio Geográfico del Ejército.

1897-1900. “Provincia de Huelva”, por Instituto Geográfico Estadístico. Cartoteca Histórica del Servicio Geográfico del Ejército.

S. XIX. "Provincia de Huelva”. Anónimo. Sin proyección, ni escala. Escala estimada 1: 100.000. Cartoteca Histórica del Servicio Geográfico del Ejército.

S. XIX. "Parte Sudoeste de la Provincia de Huelva”, Cuerpo de E. M. S. XIX ó 1870/1878 Parte de la Provincia de Huelva, Anónimo. Cartoteca Histórica del Servicio Geográfico del Ejército.

1902. “Mapa de España (Ibáñez de Ibero, 1902)”. Escala: 1:500.000. Mapa de España formado por el Excmo. señor Mariscal de Campo D. Carlos Ibáñez é Ibáñez de Ibero con motivo de la división del territorio en zonas militares para situar las reservas y depósitos del ejército (sic). Publicado en 1884 y reproducido en 1902. Lit. Instituto Geográfico y Estadístico. Sin proyección, meridiano en el origen el de Madrid. Sin escala gráfica. Cartoteca del Instituto Geográfico Nacional.

1913. "Las costas de la provincia de Huelva y sus variaciones en el periodo histórico". Autor: Maximino San Miguel de la Cámara. Bol. R. Soc. Esp. Hist. Nat. XIII: 434468. 